Sección Básica / Basic

Artículo de investigación / Research paper

\title{
Hongos asociados a Hypothenemus hampei (Coleoptera: Curculionidae: Scolytinae) en Tabasco, México
}

\author{
Fungi associated with Hypothenemus hampei (Coleoptera: Curculionidae: Scolytinae) \\ in Tabasco, Mexico
}

\author{
MAGDIEL TORRES-DE LA CRUZ1' JOSÉ DEL CARMEN GERÓNIMO-TORRES²; \\ CARLOS FREDY ORTIZ-GARCÍA ; VICTORIA AYALA ESCOBAR; \\ MANUEL PÉREZ-DE LA CRUZ ${ }^{5}$; SILVIA CAPPELLO-GARCÍA ${ }^{6}$
}

\begin{abstract}
${ }^{1}$ Ph. D. División Académica de Ciencias Biológicas, Universidad Juárez Autónoma de Tabasco, CP 86039, Tabasco, México, magtorre@colpos.mx, https:// orcid.org/0000-0002-5030-5932. ${ }^{2}$ M. Sc. División Académica de Ciencias Biológicas, Universidad Juárez Autónoma de Tabasco, CP 86039, Tabasco, México, jc.geronimo89@hotmail.com, https://orcid.org/0000-0001-9546-3339. ${ }^{3}$ Ph. D. Colegio de Postgraduados, Campus Tabasco, CP 86500, Tabasco, México, cfortizg@gmail.com, https://orcid.org/0000-0002-6820-9252. ${ }^{4}$ M. Sc. Colegio de Postgraduados, Campus Montecillo, CP 56230, Texcoco, Estado de México, México,ayalav@colpos.mx, https://orcid.org/0000-0001-5998-6175. ${ }^{5}$ Ph. D. División Académica de Ciencias Biológicas, Universidad Juárez Autónoma de Tabasco, CP 86039, Tabasco, México, perezmandoc@hotmail.com, https://orcid.org/0000-0002-0886-3025. ${ }^{6}$ Ph. D. División Académica de Ciencias Biológicas, Universidad Juárez Autónoma de Tabasco, CP 86039, Tabasco, México, cappellogs@hotmail.com, https://orcid.org/0000-0003-1354-6304.
\end{abstract}

Autor para correspondencia: Manuel PérezDe la Cruz, Ph. D. División Académica de Ciencias Biológicas, Universidad Juárez Autónoma de Tabasco, carretera VillahermosaCárdenas km 0.5, CP 86039, Tabasco, México, perezmandoc@hotmail.com, https://orcid. org/0000-0002-0886-3025

Citación sugerida / Suggested citation: TORRES-DE LA CRUZ, M.; GERÓNIMOTORRES, J. D. C.; ORTIZ-GARCÍA, C. F.; AYALA ESCOBAR, V.; PÉREZ-DE LA CRUZ, M.; CAPPELLO-GARCÍA, S. 2019. Hongos asociados a Hypothenemus hampei (Coleoptera: Curculionidae: Scolytinae) en Tabasco, México. Revista Colombiana de Entomología 45 (2): e7960. https://doi. org/10.25100/socolen.v45i2.7960

Recibido: 02-mar-2018

Aceptado: 12-mar-2019

Publicado: 8-ene-2020

Revista Colombiana de Entomología

ISSN (Impreso): 0120-0488

ISSN (En línea): 2665-4385

$\mathrm{http} / / /$ revistacolombianaentomologia.univalle.edu.co/

Open access

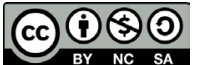

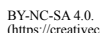

Publicadores / Publishers:

Sociedad Colombiana de Entomología

SOCOLEN (Bogotá, D. C., Colombia)

http://www.socolen.org.co

Universidad del Valle (Cali, Colombia)

http://www.univalle.edu.co/

(C) 2019 Sociedad Colombiana de Entomología - SOCOLEN y Universidad del Valle - Univalle
Resumen: En el estado de Tabasco, México, la broca del café Hypothenemus hampei, se registró por primera vez en 2004. Desde entonces se ha convertido en la principal limitante insectil de la producción de café. El objetivo del presente trabajo fue identificar la micobiota asociada a adultos de $H$. hampei, recolectados en plantaciones de café en tres municipios de Tabasco: Teapa, Tacotalpa y Huimanguillo. Se recolectaron cerezas infestadas con la broca y se extrajeron los adultos de H. hampei. Los hongos que crecieron sobre los cadáveres de este Scolytinae se aislaron e identificaron morfológicamente. Dos aislamientos de cada especie morfológica se identificaron molecularmente. Se obtuvo un total de 74 aislamientos de la cutícula de $H$. hampei, de los cuales, 52 presentaron afinidad morfológica con Fusarium solani, cinco con $F$. oxysporum, cinco con $F$. verticillioides, uno con Geotrichum candidum y 11 con Beauveria bassiana. Las secuencias de ADN mostraron similitud con secuencias del GenBank, con homologías de 99 y $100 \%$, las cuales coincidieron con cada especie morfológica. La especie más abundante fue $F$. solani $(70,2 \%)$, seguida de $B$. bassianna $(14,8 \%)$. Las especies $F$. verticillioides y $F$. oxysporum representaron cada una el $6,7 \%$. La menos abundante fue $G$. candidum $(1,3 \%)$. Los hallazgos de $F$. verticillioides y $G$. candidum son nuevos registros de hongos micromicetos asociados a H. hampei.

Palabras clave: Broca del café, Beauveria bassiana, Fusarium, Geotrichum, control biológico, Coleoptera, Curculionidae, región Neotropical.

Abstract: In the state of Tabasco, Mexico, the coffee berry borer Hypothenemus hampei, was reported for the first time in 2004. Since then it has become the main insect pest of coffee in this area. The aim of the present study was to document the mycobiota associated to H. hampei adults in coffee plantations of three localities of Tabasco: Teapa, Tacotalpa and Huimanguillo. Coffee berries infested with the coffee berry borer were collected from the field and adults of $H$. hampei were extracted. The fungi that grew on the Scolytinae cadavers were isolated and identified using taxonomic keys. Two isolates of each morphological species were molecularly identified. A total of 74 isolates was obtained from the cuticle of $H$. hampei. Of them, 52 presented morphological affinity with Fusarium solani, five with F. oxysporum, five with $F$. verticillioides, one with Geotrichum candidum and 11 with Beauveria bassiana. The DNA sequences showed similarity with sequences found in GenBank, with homologies of 99 $\%$ and $100 \%$, which coincided with each morphological species. The most abundant species was $F$. solani $(70.2 \%)$, followed by B. bassiana (14.8\%). The species $F$. verticillioides and $F$. oxysporum represented each one, at $6.7 \%$, respectively. The least abundant was $G$. candidum $(1.3 \%)$. The findings of the species $F$. verticillioides and $G$. candidum are new reports of micromycetes fungi associated with $H$. hampei. This study contributes to the knowledge of the diversity of fungi associated with $H$. hampei.

Keywords: Coffee berry borer, Beauveria bassiana, Fusarium, Geotrichum, biocontrol, Coleoptera, Curculionidae, Neotropical region.

\section{Introducción}

Hypothenemus hampei (Ferrari, 1867) (Coleoptera: Curculionidae: Scolytinae), es el insecto plaga más perjudicial en muchos países productores de café (Coffea spp.) (Vega et al. 2015; Infante 2018). Este insecto es originario de África Central y fue 
introducido a Brasil en 1913, de donde se dispersó a los demás países productores de café de América y el Caribe (Infante et al. 2014). En 1978, la broca del café ingresó a México desde Guatemala a través del Soconusco, Chiapas (Baker 1984), dispersándose a todas las áreas productoras de café en el país. En el estado de Tabasco, H. hampei se registró por primera vez en el año 2004 (Ramírez-Del Ángel et al. 2007) y se ha convertido en el principal insecto plaga del café en la entidad.

Hypothenemus hampei utiliza los frutos de café como hábitat y para alimentación de estados inmaduros y adultos (Le Pelley 1968). Para ello, las hembras adultas perforan el fruto del café y colocan sus huevos en el endospermo. Cuando nacen las larvas se alimentan de la semilla del café, provocando disminución del rendimiento hasta de un $50 \%$ (Mathieu et al. 1999). La broca del café pasa la mayor parte de su vida dentro del fruto de café, lo cual dificulta su control (Murphy y Moore 1990).

Muchas especies de Scolytinae presentan relaciones mutualistas con hongos, los cuales les sirven de alimento (Nakashima 1971). En otros casos, los hongos asociados se comportan como patógenos para la planta hospedera del insecto o pueden ser patógenos del insecto plaga (Posada et al. 1993). En Chiapas, México, Pérez et al. (2003), reportaron 15 hongos asociados a $H$. hampei; así también, Carrión y Bonet (2004) registraron 11 especies asociadas a H. hampei en plantaciones de café del estado de Veracruz. En Brasil, Gama et al. (2006), reportaron 10 géneros de hongos asociados con H. hampei.

El estudio de los hongos asociados a H. hampei contribuye al conocimiento de la biodiversidad y en el caso de hongos entomopatógenos podría resultar en una alternativa sustentable para el control de esta plaga. Al respecto, Bustillo et al. (1999) registraron a Paecilomyces lilacinus (Samson, 1974) atacando a H. hampei. Carrión y Bonet (2004) reportaron a Beauveria bassiana (Balsamo, 1835) asociada a la broca del café, y este hongo se considera el principal controlador de la broca del café (Bustillo 2004). Debido a que la micobiota de la broca del café no ha sido estudiada en Tabasco, el objetivo de la presente investigación fue identificar los hongos asociados a la cutícula de los adultos de $H$. hampei, recolectados en agroecosistemas de café del estado de Tabasco, México.

\section{Materiales y métodos}

Los sitios de muestreo se ubicaron en los tres municipios que concentran la producción de café en el área: Teapa, Tacotalpa y Huimanguillo. En cada municipio se establecieron seis sitios de muestreo (Tabla 1). El muestreo se realizó de octubre a diciembre de 2015. En cada parcela se recolectaron 100 cerezas brocadas. Las cerezas se disecaron para buscar y recolectar todos los adultos (vivos y muertos) en el interior de la cereza.

Obtención de aislamientos. Las brocas que se extrajeron de las cerezas se desinfestaron superficialmente en una solución de hipoclorito de sodio al $0,5 \%$, durante $5 \mathrm{~min}$, y se enjuagaron tres veces con agua destilada estéril. Posteriormente, los insectos se colocaron individualmente en cámara húmeda a $25^{\circ} \mathrm{C}$, durante 8 días, para permitir la expresión de hongos sobre los cadáveres. Los hongos que se desarrollaron sobre la cutícula de $H$. hampei, se aislaron y se multiplicaron en medio de cultivo agar-dextrosa de Sabouraud $+0,1 \%$ de extracto de levadura (ADS + EL) (Feng-Yan y Quing-Tao 1991).

Caracterización morfológica. Las especies de hongos se identificaron con base en las estructuras reproductivas, según Samson (1981), Humber (1997), Watanabe (2002) y el manual de laboratorio de Fusarium de Leslie y Summerell (2006). Para ello, los aislamientos se crecieron en cajas de Petri con medio ADS + EL durante $14 \mathrm{~d}$, para determinar las características de la colonia: tamaño, forma y conidiación. Además, una suspensión de esporas fue inoculada sobre discos

Tabla 1. Sitios de muestreo y aislamientos de hongos obtenidos a partir de la cutícula de Hypothenemus hampei, en plantaciones de café de Tabasco, México.

\begin{tabular}{|c|c|c|c|c|c|}
\hline Municipio & Localidad & \multicolumn{2}{|c|}{ Georreferenciación } & \multirow{2}{*}{$\begin{array}{c}\begin{array}{c}\text { Altitud } \\
\text { (m) }\end{array} \\
46\end{array}$} & \multirow[t]{2}{*}{ Aislamientos obtenidos } \\
\hline \multirow{6}{*}{ Huimanguillo } & $\mathrm{R} / \mathrm{a}$. Arena $1^{\mathrm{a}}$ sección & $\mathrm{N} 17^{\circ} 24^{\prime} 11^{\prime \prime}$ & O093³3’06”' & & \\
\hline & R/a. Pedregalito $1^{\mathrm{a}}$ sección & $\mathrm{N} 17^{\circ} 27^{\prime} 35^{\prime \prime}$ & O093³2’48” & 59 & $\mathrm{Hh} 7, \mathrm{Hh} 8, \mathrm{Hh} 9$ \\
\hline & Ejido Chimalapa $2^{\mathrm{a}}$ sección & $\mathrm{N} 17^{\circ} 24^{\prime} 43^{\prime \prime}$ & O093³7’47”' & 91 & Hh10, Hh11, Hh12, Hh13, Hh14 \\
\hline & Francisco J. Mujica & $\mathrm{N} 17^{\circ} 22^{\prime} 31^{\prime \prime}$ & O093³8'49”' & 407 & Hh15 \\
\hline & Malpasito & $\mathrm{N} 17^{\circ} 20^{\prime} 23^{\prime \prime}$ & O0933ㅜ웅' & 238 & Hh16 \\
\hline & Chimalapa & $\mathrm{N} 17^{\circ} 26^{\prime} 52^{\prime \prime}$ & O0933'ㄱ' & 203 & Hh17, Hh18, Hh19, Hh20 \\
\hline \multirow{6}{*}{ Tacotalpa } & Ejido Tapijulapa & $\mathrm{N} 17^{\circ} 27^{\prime} 30^{\prime \prime}$ & $\mathrm{O} 092^{\circ} 45^{\prime} 41^{\prime \prime}$ & 200 & $\begin{array}{l}\text { Hh21, Hh22, Hh23, Hh24, Hh25, Hh26, Hh27, Hh28, } \\
\text { Hh29, Hh30, Hh31, Hh32, Hh33 }\end{array}$ \\
\hline & $\begin{array}{l}\text { Ejido Cerro Blanco } 4^{\mathrm{a}} \\
\text { sección }\end{array}$ & $\mathrm{N} 17^{\circ} 25^{\prime} 60^{\prime \prime}$ & $\mathrm{O} 092^{\circ} 48^{\prime} 52^{\prime \prime}$ & 456 & Hh34, Hh35, Hh36, Hh37, Hh38, Hh39, Hh40 \\
\hline & $\begin{array}{l}\text { Ejido Cerro Blanco } 2^{\mathrm{a}} \\
\text { sección }\end{array}$ & $\mathrm{N} 17^{\circ} 25^{\prime} 04^{\prime \prime}$ & $\mathrm{O} 092^{\circ} 48^{\prime} 17^{\prime \prime}$ & 371 & $\begin{array}{l}\text { Hh41, Hh42, Hh43, Hh44, Hh45, Hh46, Hh47, Hh48, } \\
\text { Hh49, Hh50 }\end{array}$ \\
\hline & $\begin{array}{l}\text { Ejido Cerro Blanco } 5^{\mathrm{a}} \\
\text { sección }\end{array}$ & $\mathrm{N} 17^{\circ} 26^{\prime} 55^{\prime \prime}$ & O09249'16” & 194 & Hh51, Hh52, Hh53, Hh54, Hh55, Hh56, Hh57 \\
\hline & Ejido Palo Quemado & $\mathrm{N} 17^{\circ} 25^{\prime} 08^{\prime \prime}$ & O09250’09” & 947 & Hh58, Hh59 \\
\hline & Ejido Zonu y Pastatal & $\mathrm{N} 17^{\circ} 28^{\prime} 08^{\prime \prime}$ & O09249’09” & 188 & Hh60, Hh61, Hh62, Hh63, Hh64, Hh65 \\
\hline \multirow{3}{*}{ Teapa } & Vicente Guerrero & $\mathrm{N} 17^{\circ} 31^{\prime} 42^{\prime \prime}$ & $\mathrm{O} 092^{\circ} 55^{\prime} 57^{\prime \prime}$ & 93 & Hh66, Hh67 \\
\hline & Arcadio Zentella & $\mathrm{N} 17^{\circ} 31^{\prime} 56^{\prime \prime}$ & O09251'36" & 45 & Hh68, Hh69, Hh70, Hh71 \\
\hline & Nicolás Bravo $2^{\mathrm{a}}$ sección & $\mathrm{N} 17^{\circ} 29^{\prime} 46^{\prime \prime}$ & O09257’27’' & 473 & Hh72, Hh73, Hh74 \\
\hline
\end{tabular}


de $\mathrm{ADS}+\mathrm{EL}$ de $0,5 \mathrm{~cm}$ de diámetro, bajo un cubre objetos, para obtener estructuras microscópicas de cada aislamiento. Todos los discos inoculados se incubaron a $25^{\circ} \mathrm{C}$ en cámara húmeda durante $5 \mathrm{~d}$. Las características microscópicas de conidióforos, conidios y clamidosporas se observaron bajo el microscopio de campo claro y foto documentadas mediante digitalización de imágenes. La morfometría se realizó con el software Image Tool ${ }^{\circledR}$ (Wilcox et al. 2002). Los aislamientos "voucher" o comprobantes se depositaron en la colección de hongos entomopatógenos de la Universidad Juárez Autónoma de Tabasco (UJAT), en Villahermosa, Tabasco. La sinonimia aceptada está basada en la información ofrecida en Index Fungorum (2018), MycoBank (2018) y EOL (2018).

Caracterización molecular. Dos aislamientos de cada especie morfológica se caracterizaron molecularmente. El ADN de los hongos estudiados se obtuvo de acuerdo con Ahrens y Seemüller (1992). La amplificación de las regiones ITS1 e ITS2 de los genes ribosomales (RNAr) se efectuó mediante la técnica de PCR, utilizando los iniciadores universales ITS4 (TCC TCC GCT TAT TGA TAT GC) e ITS5 (GGA AGT AAA AGT CGT AAC AAG G) (White et al. 1990).

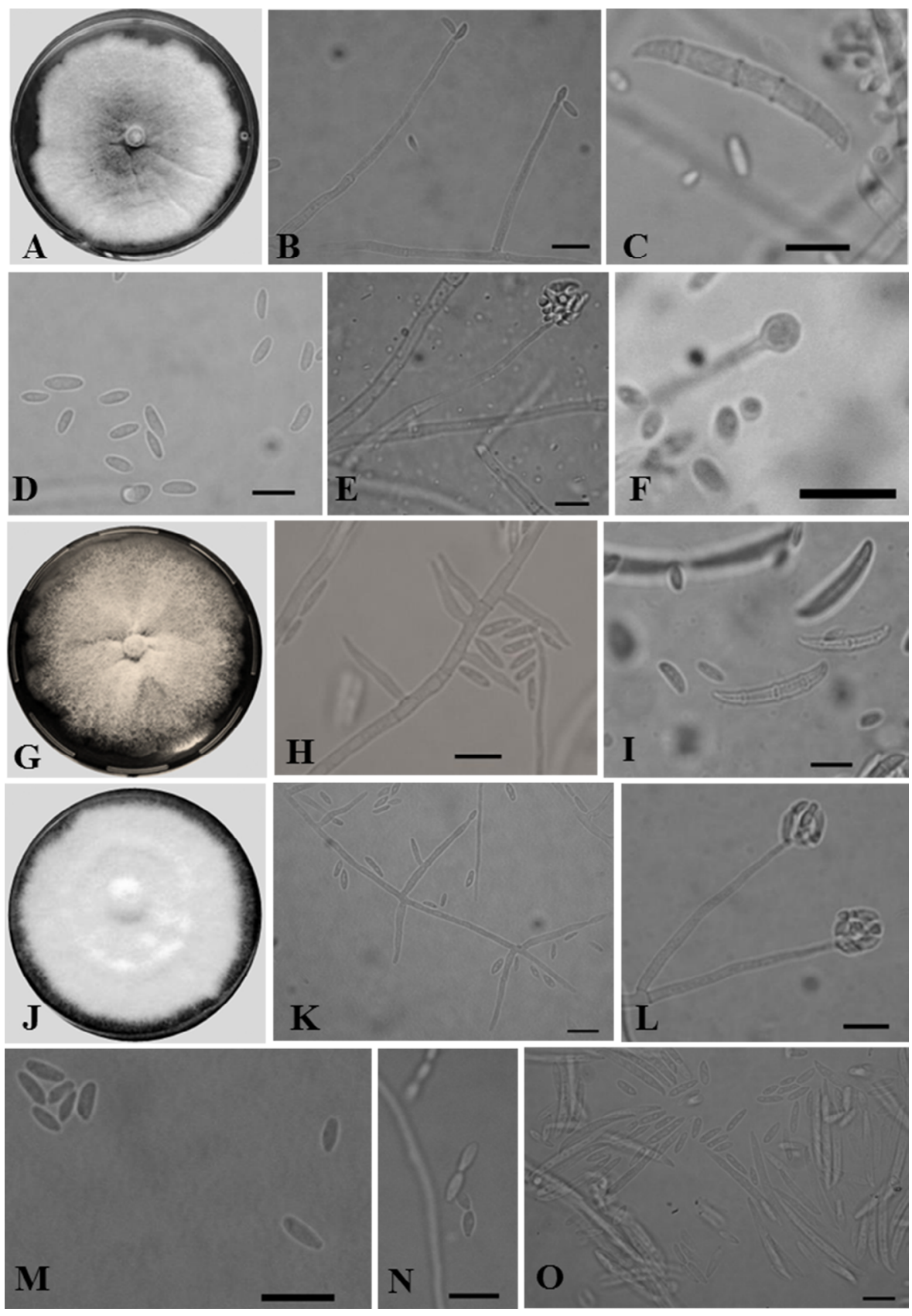

Figura 1. Hongos asociados a la cutícula de Hypothenemus hampei, en medios de cultivo PDA, a $25^{\circ} \mathrm{C}$. A. Colonia de $F$. solani; B, C, D, E y F. Conidióforo, macroconidios, microconidios, falsas cabezas y clamidospora terminal de $F$. solani, respectivamente. G. Colonia de $F$. oxysporum. H, I. Conidióforos cortos, microconidios y macroconidios de F. oxysporum. J. Colonia de F. verticillioides. $\mathbf{K}, \mathbf{L}, \mathbf{M}, \mathbf{N}, \mathbf{O}$. Conidióforos, falsas cabezas, microconidios, microconidios en cadenas, macroconidios, respectivamente, de F. verticillioides. Descripción: Leslie y Summerell (2006). Barra $=10 \mu \mathrm{m}$. 

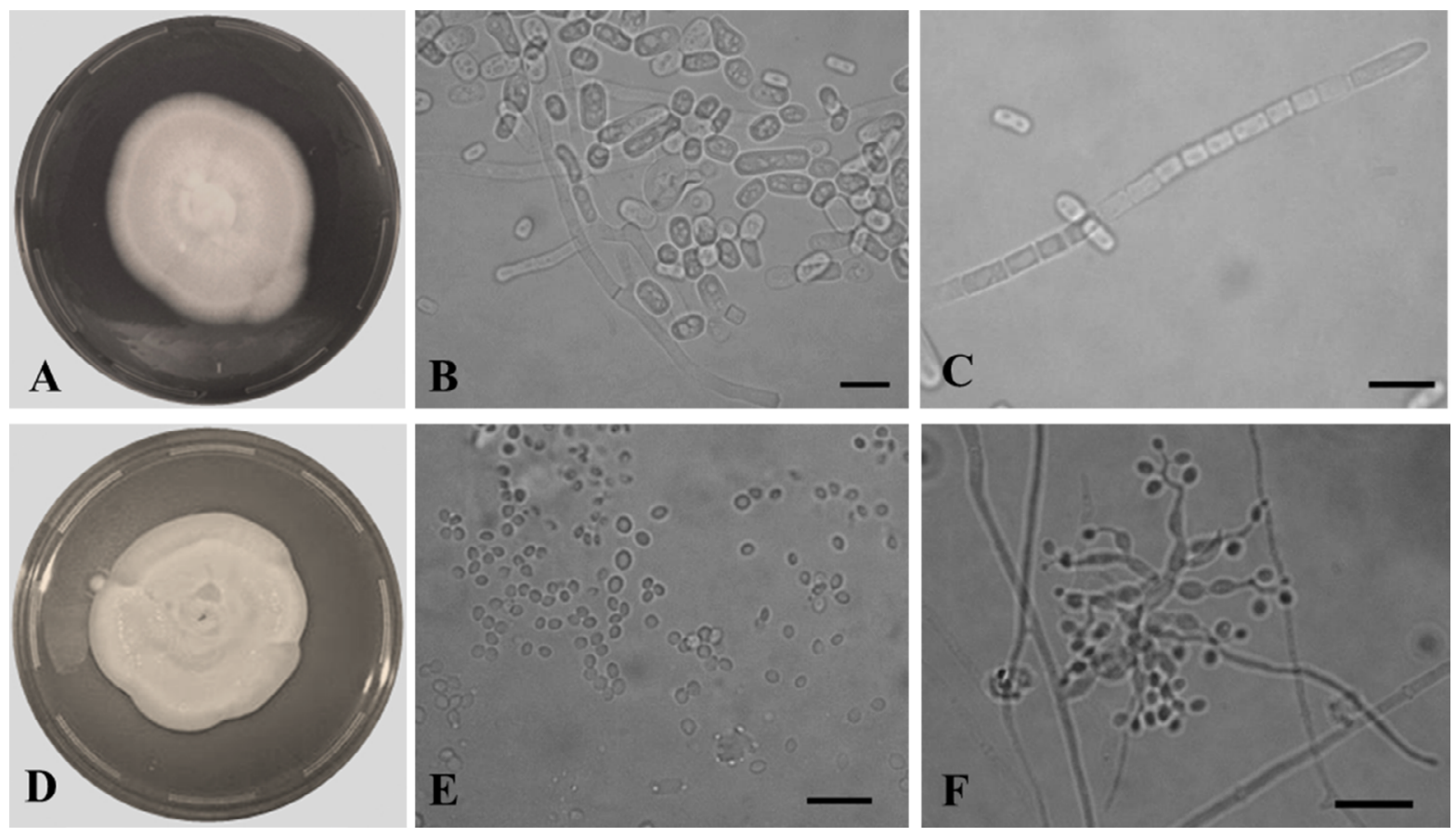

Figura 2. Hongos de los géneros Geotrichum y Beauveria, asociados a la cutícula de Hypothenemus hampei, en medio de cultivo PDA. A. Colonia. B. Artrosporas. C. Hifas de G. candidum dividida en cadena de artroconidios; descripción: Watanabe (2002). D. Colonia. E. Conidios. F. Conidióforos de B. bassiana; descripción: Samson (1981) y Humber (1997). Barra $=10 \mu \mathrm{m}$.

La amplificación y visualización de los productos finales se realizó según el protocolo de Ahrens y Seemüller (1992). Los productos amplificados se purificaron con el kit Wizard (Promega ${ }^{\circledR}$ ) y se enviaron para secuenciación a Macrogene ${ }^{\circledR}$. Las secuencias se compararon con la base de datos del banco de genes del National Center for Biotechnology Information (NCBI 2015). Para la comparación de los valores generados con la secuencia de estudio se consideró una similitud $\geq 97 \%$ (Stackebrandt y Goebel 1994).

\section{Resultados}

Un total de 74 aislamientos de micromicetos fue obtenido de la cutícula de $H$. hampei. De ellos, 52 presentaron afinidad morfológica con Fusarium solani (Martinus) Saccardo, 1881; cinco con F. oxysporum (Schlechtendahl) Snyder y Hansen,

Tabla 2. Especies de hongos micromicetos aislados a partir de la cutícula de Hypothenemus hampei, identificados por taxonomía clásica.

\begin{tabular}{lc}
\hline \multicolumn{1}{c}{ Aislamiento } & Especie morfológica \\
\hline Hh6, Hh10, Hh11, Hh12, Hh13, Hh14, & \\
Hh15, Hh17, Hh18, Hh21, Hh22, Hh23, & \\
Hh24, Hh25, Hh26, Hh27, Hh28, Hh29, & \\
Hh30, Hh31, Hh32, Hh33, Hh34, Hh35, & Fusarium solani \\
Hh36, Hh38, Hh41, Hh43, Hh44, Hh45, & \\
Hh49, Hh50, Hh51, Hh53, Hh54, Hh55, & \\
Hh56, Hh57, Hh58, Hh59, Hh60, Hh61, & \\
Hh62, Hh63, Hh64, Hh66, Hh67, Hh70, & \\
Hh71, Hh72, Hh73, Hh74 & Fusarium oxysporum \\
Hh37, Hh39, Hh42, Hh68, Hh69 & Fusarium verticillioides \\
Hh5, Hh19, Hh47, Hh48, Hh52 & Geotrichum candidum \\
Hh20 & Beauveria bassiana \\
Hh1, Hh2, Hh3, Hh4, Hh7, Hh8, Hh9, Hh16, \\
Hh40, Hh46, Hh65
\end{tabular}

1940; cinco con F. verticillioides (Saccardo) Nirenberg, 1976, (Fig. 1); uno con Geotrichum candidum (Link, 1809) y 11 con Beauveria bassiana (Balsamo) Vuillemin, 1912 (Fig. 2, Tabla 2).

La región amplificada de los aislamientos $\mathrm{Hh} 7$ y H16, especie morfológica $B$. bassiana, presentó un tamaño de 581 $\mathrm{pb}$. La región de los aislados H12 y H61, especie morfológica F. solani, obtuvo un tamaño de $568 \mathrm{pb}$. Así también, las cepas Hh37 y Hh69, especie morfológica $F$. oxysporum, mostraron un tamaño de $556 \mathrm{pb}$. Las cepas Hh19 y Hh48 obtuvieron 563 pb y el aislamiento Hh20, especie morfológica G. candidum, presentó $361 \mathrm{pb}$. Las secuencias de estudio mostraron similitud con secuencias del GenBank, con homologías del 99 y 100 $\%$, las cuales coincidieron con especies correspondientes a la especie morfológica, confirmando que los hongos aislados de la cutícula de $H$. hampei corresponden a cinco especies mencionadas (Tabla 3).

Tabla 3. Especies de hongos presentes en la cutícula de Hypothenemus hampei, determinados por amplificación de los espaciadores transcritos internos (ITS) 1 y 2 , y de la región $5.8 \mathrm{~S}$ del ADN ribosomal.

\begin{tabular}{clcc}
\hline Cepa & $\begin{array}{c}\text { Identificación } \\
\text { morfológica y molecular }\end{array}$ & $\begin{array}{c}\text { Homología } \\
(\%)\end{array}$ & $\begin{array}{c}\text { Cepa de } \\
\text { referencia } \\
\text { (GenBank) }\end{array}$ \\
\hline Hh7 & Beauveria bassiana & 100 & JQ266172 \\
Hh16 & Beauveria bassiana & 99 & JQ320361 \\
Hh12 & Fusarium solani & 99 & JQ723750 \\
Hh61 & Fusarium solani & 99 & KX078455 \\
Hh37 & Fusarium oxysporum & 99 & KJ715962 \\
Hh69 & Fusarium oxysporum & 99 & EU839381 \\
Hh19 & Fusarium verticillioides & 99 & KP003945 \\
Hh48 & Fusarium verticillioides & 99 & KX196811 \\
Hh20 & Geotrichum candidum & 99 & KJ579946 \\
\hline
\end{tabular}


La especie más abundante fue $F$. solani $(70,2 \%)$, seguida de B. bassiana (14,8 \%). Fusarium verticillioides y F. oxysporum representaron cada una el 6,7\% respectivamente. La especie menos abundante fue G. candidum (1,3\%) (Fig. 3).

\section{Discusión}

Este trabajo se constituye el primer estudio de los hongos micromicetos asociados a la cutícula de $H$. hampei a partir de brocas recolectadas en los municipios que concentran la producción de café en Tabasco. En plantaciones de café del estado de Chiapas, Pérez et al. (2003) reportaron 15 especies fúngicas en las que se incluye $F$. solani. Así también, Carrión y Bonet (2004) en Veracruz encontraron 11 especies, en las que se incluyen $F$. solani. F. oxysporum y B. bassiana. Para Brasil, Gama et al. (2006), registraron 10 géneros de hongos asociados con $H$. hampei, en las que se mencionan Fusarium, Geotrichum y Beauveria, los cuales también son reportados en la presente investigación. En nuestro estudio, la abundancia de especies encontradas sobre $H$. hampei fue menor a la encontrada por otros autores, lo cual pudo obedecer a diferencia de los métodos utilizados. También es importante señalar que, en esta investigación, el muestreo se realizó solamente en la época de lluvias, por lo que la micobiota asociada a $H$. hampei puede variar con respecto a otras épocas del año.

De las especies aisladas sobre H. hampei, F. solani fue la especie más abundante, lo cual fue similar a lo encontrado por Pérez et al. (2003) en el estado de Chiapas y por Gama et al. (2006), en Brasil. Morales et al. (2000), reportaron que $F$. solani mantiene una relación simbiótica mutualista con $H$. hampei; sin embargo, Pérez et al. (2005) concluyeron que no existe dicha relación. Fusarium solani también ha sido descrito como causante de la muerte descendente de ramas de café (Wellman 1977). Esta especie causa diversos tipos de enfermedades en al menos 111 especies de 87 géneros de plantas (Kolattukudy y Gamble 1995). Otros autores lo han descrito como parásito de diferentes insectos (Feng-Yan y Quing-Tao 1991) y como agente causal de enfermedades en humanos (Hernández et al. 2011).

Del género Fusarium también se identificaron las especies $F$. oxysporum y $F$. verticillioides. La presencia de F. oxysporum asociada a $H$. hampei coincide con los

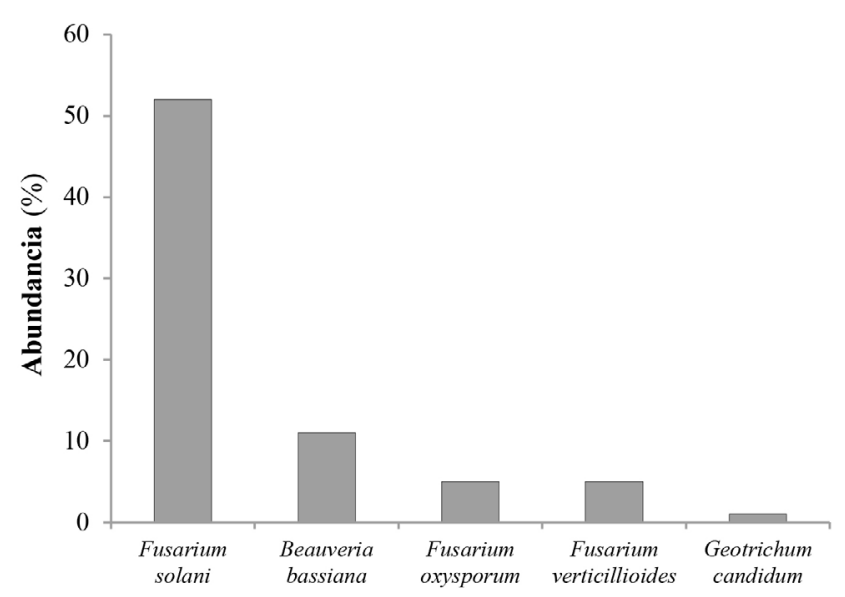

Figura 3. Abundancia relativa de micromicetos asociados a la cutícula de adultos de Hypothenemus hampei en plantaciones de café de Tabasco, México. resultados de Carrión y Bonet (2004), en plantaciones de café de Veracruz. De acuerdo con estos autores, la presencia de $F$. oxysporum y $F$. solani en la cutícula de $H$. hampei y en sus galerías, puede tener repercusiones en la salud de la planta. Al respecto, Wellman (1977) reportó a $F$. oxysporum como agente causal de marchitez en plantas de café. $F$. oxysporum es cosmopolita y parasita más de 100 especies de plantas, gimno y angiospermas, en diferentes formas especiales (Bosland 1988). Otros autores lo han descrito como parásito de insectos (Sumling et al. 2013; Feng-Yan y Quing-Tao 1991). Por su parte, F. verticillioides no había sido asociado a $H$. hampei; sin embargo, se le ha reportado como entomopatógeno de Tropidacris collaris y Ronderosia bergi (Orthoptera: Acridoidea) (Pelizza et al. 2010). Por otro lado, F. verticillioides es considerado el principal patógeno de Zea mays, ya que es capaz de provocar pudriciones en la raíz, el tallo y la mazorca (Desjardins 2006).

Geotrichum candidum fue la especie menos abundante y no había sido reportada asociada a $H$. hampei; aunque Gama et al. (2006), habían registrado al género asociado a la broca. Así también, se tienen reportes de G. candidum como patógeno de plantas, causando pudrición en frutas y hortalizas (Trigos et al. 2008) aunque se desconoce su efecto sobre las cerezas de café. Por otro lado, G. candidum, agente causal de la geotricosis en humanos (Caretta 1959), no presenta registro alguno como entomopatógeno. Esta especie y $F$. verticillioides se suman a la lista de micromicetos asociados a $H$. hampei, junto con aquellos reportados por Pérez et al. (2003) y Carrión y Bonet (2004).

Beauveria bassiana fue la especie más abundante, después de $F$. solani y se encontró en el $40 \%$ de los sitios muestreados. B. bassiana es el hongo entomopatógeno más utilizado en el control biológico de H. hampei. Al respecto, Bustillo (2004) reportó que, bajo condiciones de campo, B. bassiana $\left(1 \times 10^{9}\right.$ conidios/árbol) mostró el $30 \%$ de efectividad sobre la broca, a los cinco días después de la aplicación. Por otro lado, $B$. bassiana es usado para el control de otros insectos plaga y es la especie de entomopatógeno más utilizada en el mundo (Alves et al. 2003).

\section{Conclusiones}

Se identificaron cinco especies de hongos asociados a cadáveres de $H$. hampei. Cuatro de ellas registradas como especies fitopatógenas de diferentes cultivos de las cuales, $F$. solani y $F$. oxysporum se han reportado como fitopatógenos del café y otras plantas tropicales. Las tres especies de Fusarium, identificadas en esta investigación, han sido descritas como parásitos de otros insectos; sin embargo, se desconoce el papel que desempeñan sobre $H$. hampei. La abundancia de especies asociadas a $H$. hampei, obtenida en cámaras húmedas, fue menor a la de otros autores. Las especies $F$. verticillioides y G. candidum son nuevos reportes de hongos micromicetos asociados a $H$. hampei. Se detectó la presencia de $B$. bassiana parasitando a $H$. hampei en el $40 \%$ de los sitios de muestreo. Este estudio es un aporte al conocimiento de la diversidad de hongos asociados a H. hampei.

\section{Agradecimientos}

Este trabajo fue financiando por el Programa de Fomento a la Investigación de la Universidad Juárez Autónoma de Tabasco, clave: UJAT-2012-IB-02. 


\section{Literatura citada}

AHRENS, U.; SEEMÜLLER, E. 1992. Detection of DNA of plant pathogenic mycoplasmalike organisms by a polymerase chain reaction that amplifies a sequence of the $16 \mathrm{~S}$ rDNA gene. Phytopathology 82 (1): 828-832. https://doi.org/10.1094/ Phyto-82-828

ALVES, S. B.; PEREIRA, R. M.; LOPES, R.; TAMAI, M. B. 2003. Use of entomopathogenic fungi in latin America. pp. 193-211. En: Upashyay, R. K. (Ed.). Advances in microbial control of insec pest. Kluwer Academic. New York. 340 p. https://doi. org/10.1007/978-1-4757-4437-8_11

BAKER, P. S. 1984. Some aspects of the behavior of the coffee berry borer in relation to its control in southern Mexico (Coleoptera, Scolytidae). Folia Entomológica Mexicana 61: 9-24.

BOSLAND, P. W. 1988. Fusarium oxysporum a pathogen of many plant species. Advances in Plant Pathology 6 (1): 281-289. https://doi.org/10.1016/B978-0-12-033706-4.50023-2

BUSTILLO, A. E.; BERNAL, M. G.; CHÁVEZ, B.; BENAVIDES, P. 1999. Dynamics of Beauveria bassiana and Metarhizium anisopliae infecting Hypothenemus hampei (Coleoptera: Scolytidae) populations emerging from fallen coffee berries. Florida Entomologist 82 (4): 49-498. https://doi. org/10.2307/3496468

BUSTILlO, P. A. E. 2004. ¿Cómo participa el hongo Beauveria bassiana en el manejo integrado del café? Brocarta Cenicafé (Colombia) 37 (1): 1-4.

CARETTA, G. 1959. Caratteristiche morfo-biologiche di ceppi fungini del genere Geotrichum isolati da materiale umano. Mycopathologia 11 (3): 217-237. https://doi.org/10.1007/ BF02063079

CARRIÓN, G.; BONET, A. 2004. Mycobiota associated with the coffee berry borer (Coleoptera: Scolytidae) and its galleries in fruit. Annals of the Entomological Society of America 97 (3): $492-$ 499. https://doi.org/10.1603/0013-8746(2004)097[0492:MAWT $\mathrm{CB}] 2.0 . \mathrm{CO} ; 2$

DESJARDINS, A. E. 2006. Fusarium mycotoxins: chemistry, genetics and biology. American Phytophatological Society, St. Paul, MN. 268 p.

EOL - ENCYCLOPEDIA OF LIFE. 2018. Trichoderma reesei E. G. Simmons 1977. Disponible en: http://eol.org/pages/192915/ names. [Fecha revisión: 20 enero 2018].

FENG-YAN, B.; QUING-TAO, C. 1991. Fusarium species on some insects from China. Acta Mycologica Sinica 10 (1): 120-128.

GAMA, F.; TEIXEIRA, C.; GARCIA, A.; COSTA, J.; LIMA, D. 2006. Diversidade de fungos filamentosos associados a Hypothenemus hampei (Ferrari) (Coleoptera: Scolytidae) e suas galerias em frutos de Coffea canephora (Pierre). Neotropical Entomology 35 (5): 573-578. https://doi.org/10.1590/S1519566X2006000500002

HERNÁNDEZ, C. C.; NÚÑEZ, Q. A.; RODRÍGUEZ, F. Y.; CARNOT, U. J.; MUÑIO, P. J.; PÉREZ, R. G.; CHÁVEZ, M. R.; MOYA, P. I.; LLANES, R. N.; HART, C. M. 2011. Sepsis sistémica por Fusarium solani en pacientes con leucemias agudas. Reporte de dos casos. Revista de Hematología Mexicana 12 (4): 287-292.

HUMBER, R. 1997. Fungi: identification. pp. 153-185. En: Lacey, L. (Ed.). Manual of techniques insect pathology. Academic press, New York, EE. UU. 369 p. https://doi.org/10.1016/B978012432555-5/50011-7

INDEX FUNGORUM. 2018. Disponible en: www.indexfungorum. org/ [Fecha revisión: 18 enero 2018].

INFANTE, F. 2018. Pest management strategies against the coffee berry borer (Coleoptera: Curculionidae: Scolytinae). Journal of Agricultural and Food Chemistry 66 (21): 5275-5280. https:// doi.org/10.1021/acs.jafc.7b04875

INFANTE, F.; PÉREZ, J.; VEGA, F. E. 2014. The coffee berry borer: the centenary of a biological invasion in Brazil. Brazilian Journal of Biology 74 (3): 125-126. https://doi.org/10.1590/1519-6984.15913
KOLATTUKUDY, P. E.; GAMBLE, D. L. 1995. Nectria haematococca: pathogenesis and host specificity in plant diseases. pp. 83-102. En: Singh, U. S.; Singh, R. P.; Kohmoto, K. (Eds.). Pathogenesis and host specificity in plant pathogenic fungi and nematodes, Vol. 2 Eukaryotes. Pergamon, Oxford. 300 p.

LE PELLEY, R. H. 1968. Pests of coffee. Longmans Green and Co., Londres. 590 p.

LESLIE, J. F.; SUMMERELL, B. A. 2006. The Fusarium laboratory manual. Blackwell Publishing Professional. EE. UU. 388 p. https://doi.org/10.1002/9780470278376

MATHIEU, F.; BRUN, L. O.; FRÉROT, B.; SUCKLING, D. M.; FRAMPTON, C. 1999. Progression in field infestation is linked with trapping of coffee berry borer, Hypothenemus hampei (Col., Scolytidae). Journal of Applied Entomology 123 (9): 535-540. https://doi.org/10.1046/j.1439-0418.1999.00400.x

MYCOBANK. Database. Disponible en: www.mycobank.org. [Fecha revisión: 20 enero 2018].

MORALES, R. J.; ROJAS, M. G.; SITTERTZ, B. H.; SALDAÑA, G. 2000. Symbiotic relationship between Hypothenemus hampei (Coleoptera: Scolytidae) and Fusarium solani (Moniliales: Tuberculariaceae). Annals of the Entomological Society of America 93 (3): 541-547. https://doi.org/10.1603/00138746(2000)093[0541:SRBHHC]2.0.CO;2

MURPHY, S. T.; MOORE, D. 1990. Biological control of the coffee berry borer, Hypothenemus hampei (Ferrari) (Coleoptera: Scolytidae): previous programmes and possibilities for the future. Biocontrol News and Information 11 (1): 107-117.

NAKASHIMA, T. 1971. Notes on the associated fungi and the mycetangia of the ambrosia beetle, Crossotarsus niponicus Blandford (Coleoptera: Platypodidae). Applied Entomology and Zoology 6 (1): 131-137. https://doi.org/10.1303/aez.6.131

NATIONAL CENTER FOR BIOTECHNOLOGY INFORMATION (NCBI). 2015. Disponible en: http://ncbi.nlm.nih.gov [Fecha revisión: 15 octubre 2015].

PELIZZA, S. A.; STENGLEIN, S. A.; CABELlO, M. N.; DINOLFO, M. I.; LANGE, C. E. 2010. First record of Fusarium verticillioides as an entomopathogenic fungus of grasshoppers. Journal of Insect Science 11 (70): 1-8. https://doi. org/10.1673/031.011.7001

PÉREZ, J.; INFANTE, F.; VEGA, E. F.; HOLGUÍN, F.; MACÍAS, J.; VALLE, J.; NIETO, G.; PETERSON, S. W.; KURTZMAN, C. P.; O'DONNELL, K. 2003. Mycobiota associated with the coffee berry borer (Hypothenemus hampei) in Mexico. Mycological Research 107 (7): 879-887. https://doi.org/10.1017/ S0953756203007986

PÉREZ, J.; INFANTE, F.; VEGA, F. E. 2005. Does the coffee berry borer (Coleoptera: Scolytidae) have mutualistic fungi? Annals of the Entomological Society of America 98: 483-490. https://doi. org/10.1603/0013-8746(2005)098[0483:DTCBBC]2.0.CO;2

POSADA, F. J.; BUSTILLO, A. E.; SALDARRIAGA, G. 1993. Primer registro del ataque de Hirsutella eleutheratorum sobre la broca del café en Colombia. Cenicafé (Colombia) 44 (4): 155-158.

RAMÍREZ-DEL ÁNGEL, M. M.; GONZÁLEZ C.; BELLO, R.; ROMERO, B. 2007. Campaña nacional contra la broca del café en México: Operación y perspectivas. pp. 73-81. En: Barrera, J. F.; García, A.; Domínguez, V.; Luna C. (Eds.). La broca del café en América tropical: Hallazgos y enfoques. Sociedad Mexicana de Entomología y El Colegio de la Frontera Sur. México. $141 \mathrm{p}$.

SAMSON, R. A. 1981. Identification the entomopathogenic Deuteromycetes. pp. 93-106. En: Burgers, H. D. (Ed.). Microbial control of pest and plant disease. Academic Press. Londres. 949 p.

STACKEBRANDT, E.; GOEBEL B. M. 1994. Taxonomic note: a place for DNA-DNA reassociation and 16S rRNA sequence analysis in the present species definition in bacteriology. International Journal of Systematic Bacteriology 44 (4): 846849. https://doi.org/10.1099/00207713-44-4-846

SUMLING, Y.; CASTILLO, J.A. B.; LEZCANO, J.; PIEPENBRING, M.; CÁCERES, O. 2013. Hongos entomopatógenos asociados 
a insectos recolectados en plantaciones de café en el oeste de Panamá. Tecnociencia 15 (2): 29-391.

TRIGOS, A.; RAMÍREZ, K; SALINAS, A. 2008. Presencia de hongos fitopatógenos en frutas y hortalizas y su relación en la seguridad alimentaria. Revista Mexicana de Micología 28 (1): 125-129.

VEGA, F. E.; INFANTE, F.; JOHNSON, A. J. 2015. The genus Hypothenemus, with emphasis on H. hampei, the coffee berry borer. pp. 427-494. En: Vega, F. E.; Hoffstetter, R. W. (Eds.). Bark beetles: Biology and ecology of native and invasive species. Academic Press. EE. UU. 620 p. https://doi.org/10.1016/B9780-12-417156-5.00011-3

WATANABE, T. 2002. Pictorial atlas of soil and seed fungi: Morphologies of cultured fungi and key to species. CRC Press. Florida. 486 p. https://doi.org/10.1201/9781420040821

WELLMAN, F. L. 1977. Dictionary of tropical American crops and their diseases. The Scarecrow Press, Inc. Nueva Jersey. 515 p.

WHITE, T.; BRUNS, T.; LEE, S.; TAYLOR, J. 1990. Amplification and direct sequencing of fungal ribosomal RNA genes for phylogenetics. pp. 315-322. En: Innis, M. A.; Gelfand, D. H.; Shinsky, J. J.; White, T. J. (Eds.). PCR Protocols: a guide to methods and applications. Academic Press. San Diego. 322 p. https://doi.org/10.1016/B978-0-12-372180-8.50042-1

WILCOX, C. D.; DOVE, S. B.; MCDAVID, W. D.; GREER, D. B. 2002. UTHSCSA Image Tool version 3.0: Freeware software available from the Department of Dental Diagnostic Science at the University of Texas Health Science Center at San Antonio, San Antonio, USA.

\section{Origen y financiamiento}

Este trabajo forma parte del proyecto "Hongos entomopatógenos asociados al agroecosistema café en Tabasco, México, y su potencial de biocontrol in vitro hacia Hypothenemus hampei" financiando por el Programa de Fomento a la Investigación de la Universidad Juárez Autónoma de Tabasco, clave: UJAT-2012-IB-02.

\section{Contribución de los autores}

Magdiel Torres-de la Cruz: Lider del estudio, se encargó de coordinar el trabajo en equipo, y conciliar las diversas contribuciones. Responsable de la identificación morfológica y molecular.

José del Carmen Gerónimo-Torres: Responsable del muestreo y aislamiento de los hongos.

Carlos Fredy Ortiz-García: Responsable de la foto documentación de las características morfológicas de los aislamientos fúngicos.

Victoria Ayala Escobar: Se encargó de la extracción de ADN y PCR.

Manuel Pérez-de la Cruz: Fue responsable de la identificación morfológica de Hypothenemus hampei.

Silvia Cappello-García: Asesora en la identificación morfológica de los hongos. 